\title{
Engineered Electrospun Poly(caprolactone)/Polycaprolactone-g- Hydroxyapatite Nano-fibrous Scaffold Promotes Human Fibroblasts Adhesion and Proliferation
}

\author{
F. Keivani ${ }^{1}$, P. Shokrollahi ${ }^{*}, 2$, M. Zandi ${ }^{2}$, S. Irani $^{1}$, F.Shokrolahi ${ }^{2}$, S.C. Khorasani ${ }^{1}$ \\ ${ }^{1}$ Biology Department, Science and Research Branch, Islamic Azad University, Tehran, Iran \\ ${ }^{2}$ Department of Biomaterials, Faculty of Science, Iran Polymer and Petrochemical Institute, Tehran, Iran
}

\begin{abstract}
:
Polycaprolactone (PCL)/ hydroxyapatite nano-composites are among the best candidates for tissue engineering. However, interactions between nHAp and PCL are difficult to control leading to inhomogeneous dispersion of the bio-ceramic particles. Grafting of polymer chains at high density/chain length while promotes the phase compatibility may result in reduced HAp exposed surface area and therefore, bioactivity is compromised. This issue is addressed here by grafting PCL chains onto HAp nano-particles through ring opening polymerization of $\varepsilon$-caprolactone (PCL-g-HAp). FTIR and TGA analysis showed that PCL (6.9 wt\%), was successfully grafted on the HAp. PCL/PCL-g-HAp nano-fibrous scaffold showed up to 10 and $33 \%$ enhancement in tensile strength and modulus, respectively, compared to those of PCL/HAp. The effects of HAp on the in vitro HAp formation was investigated for both the PCL/HAp and PCL/PCL-g-HAp scaffolds. Precipitation of HAp on the nano-composite scaffolds observed after 15 days incubation in simulated body fluid (SBF), as confirmed by scanning electron microscopy (SEM), and energy dispersive X-ray spectroscopy (EDX).

Human fibroblasts were seeded on PCL, PCL/HAp and PCL/PCL-g-HAp scaffolds. According to MTT assay, the highest cell proliferation was recorded for PCL/PCL-g-HAp nano-composite, at all time intervals (1-21 days, $\mathrm{p}<0.001$ ). Fluorescent microscopy (of DAPI stained samples) and electron microscopy images showed that all nano-fibrous scaffolds (PCL, PCL/HAp, and PCL/PCL-g-HAp), were non-toxic against cells, while more cell adhesion, and the most uniform cell distribution observed on the PCL/PCL-g-HAp.

Overall, grafting of relatively short chains of PCL on the surface of HAp nano-particles stimulates fibroblasts adhesion and proliferation on the PCL/PCL-g-HAp nano-composite.
\end{abstract}

Key words: Tissue engineering, Polycaprolactone grafted HAp, Human fibroblast cells, Electrospinning

*Corresponding author; e_mail: p.shokrolahi@ippi.ac.ir

\section{Introduction}

Even though most of tissues have a unique regenerative ability, in many cases there is a need for adjuvant therapy. This necessitates development of tissue regeneration strategies such as tissue engineering [1]. Scaffolds, cells and signaling molecules are three basic components of 
any tissue engineering approach [2]. Interconnected porous scaffolds provide a biodegradable physical space to structurally support cells attachment, growth, and proliferation and also exchange of biological molecules [3]. Focusing on bone tissue engineering, synthetic polymers such as polyglycolic acid (PLGA), poly lactic acid (PLA), and poly caprolactone (PCL), as well as their co-polymers, or natural polymers like collagen and chitosan have been combined with bioceramics such as hydroxyapatite (HAp) and tri-calcium phosphates (TCP) and studied in great details [4-6] in terms of mechanical properties, degradability and bioactivity.

In any scaffold preparation practice for tissue engineering, there is a main requirement to have interconnected pore network in order to enable oxygen, nutrients and exudates as well as scaffold degradation products transportation. So, considerable efforts have been focused on improvement of the scaffold preparation strategies with aptly designed structure and also modified surface properties [7-9]. Electrospinning is now well known as a simple and inexpensive method of scaffold fabrication [6]. With electrospinning, it became possible to produce fibrous nano-composite scaffolds with highly interconnected pores network.

Nano-composites of PCL, as a biodegradable polymer and HAp as a bioactive ceramic, have offered versatile mechanical and biological properties $[10,11]$. PCL is an FDA approved biodegradable polymer with non-toxic degradation by-products and slow degradation rate [12]. Therefore, this polymer is a good candidate for long-term implantable systems in hard and soft tissue engineering. HAp on the other hand, is a biocompatible and highly hydrophilic inorganic, which is capable to increase hydrophilicity of most of the synthetic polymers that are hydrophobic in nature. HAp also improves mechanical strength of these polymers. The most important features of hydroxyapatite are excellent osteoconductivity and good hydrophilicity. 
However, lack of sufficient interactions between inorganic hydroxyapatite and organic polymer matrices leads to inhomogeneous distribution of the particles and weak mechanical properties. This issue has been addressed by grafting polymer chains on the HAp surface taking different synthesis strategies. For example, Hong et al., grafted PLLA on HAp through ring opening polymerization and reported that tensile strength of the PLLA-g-HAp/PLLA nano-composite improved about $30 \%$ compared to that of the similar HAp/PLLA nanocomposite [12]. Recently, Liu et al., have performed surface graft polymerization of poly bisphenol A glycidyl methacrylate (poly (Bis-GMA) - PGHW), on hydroxyapatite whiskers (HW), and monitored its effect on physical and mechanical properties of the resulting (dental) composite resin. They concluded that flexural strength increased about $11 \%$ upon composite preparation with PGHW compared with that filled with native HW [13]. In an independent study Qiu et al., surface modified hydroxyapatite with L-lactic acid and subsequently carried out grafting polymerization of L-lactide on the modified surface ( $\mathrm{p}-\mathrm{HAp}$ ), and showed that the resulting p-HAp/PLLA composite exhibited superior tensile strength and modulus in comparison with HAp/PLLA composite at similar bioceramic loading [14]. Comb-shaped poly ( $\varepsilon$-caprolactone) brushes were grown on the surface of nano-hydroxyapatite (n-HAp), by combination of atom transfer radical polymerization (ATRP) and ring-opening polymerization (ROP), at a high density of about $80 \%$ by Zeng et al. [15]. Aiming to improve composite material properties, biomolecules were also immobilized on the HAp surface. In a model study, HAp was surface-modified by addition of $\beta$-alanine ( $\beta$-Ala), on which ringopening polymerization of $\gamma$-benzyl-L-glutamate-N-carboxy-anhydride (BLG-NCA) was subsequently performed, HAp-PBLG. It was shown then that particle size of HAp-PBLG had decreased significantly and that the resulting particles appeared less agglomerated relative to that of the native HAp crystals [16]. 
However, despite a rich literature on physical and mechanical properties of surface modified HAp/polymer nano-composites, effect of such surface modification strategies on biological and in particular cell-biomaterial interaction remained almost neglected.

Surface modification of HAp nano-particles with long chain organic molecules improves the nano-particles dispersability and therefore, enhances mechanical and physical properties of the polymer composites containing such particles compared to similar composites with native particles. However, such strategies may compromise the bioceramics exposed surface area and therefore result in limited bioactivity. For example, recently, Lee et al., have performed graft polymerization of epsilon-caprolactone on the surface of hydroxyapatite nano-particles (EC-HAp), and studied the mechanical and biological properties of PCL/EC-HAp nanocomposite in comparison with PCL/HAp. According to the result of that study, the composite mechanical properties improved remarkably upon surface grafting [17]. However, fibroblasts proliferation had not shown any improvement until EC-HAp loading approached $30 \mathrm{wt} \%$, which can be attributed to compromise of exposed surface area upon surface grafting [18].

Shokrollahi P., et al., have recently chemically immobilized supramolecularly functionalized short chain alkane moieties on both nano- (nHAp), and micron-sized hydroxyapatite (mHAp). Thay showed that both the dispersability and the bioactivity have improved in both the PCL/nHAp and the PCL/mHAp composites, significantly [10-11, 19]. It might be argued therefore, that the long chain PCL grafts, while improving the dispersion, may reduce exposed surface area of the HAp nano-particles effectively.

This report aims to discover effect of a relatively shorter grafting time on bioactivity of PCL/nHAp nano-composites. Therefore, the caprolactone grafting was performed for a remarkably shorter reaction time of 7 hour (much shorter than 24 hours that was reported by Lee et al., [18] at similar reaction condition, to examine if graft chain length/grafting density can modulate PCL/HAp nano-composites bioactivity. 


\section{Material and methods}

\subsection{Materials:}

Stannous octanoate $\left(\mathrm{Sn}(\mathrm{oct})_{2}\right)$ was purchased from sigma-Aldrich, Germany. $\varepsilon$-caprolactone (Acros $99 \%$, USA), was dried over calcium hydride for $48 \mathrm{~h}$, followed by vacuum distillation before use. PCL (molecular weight $(\mathrm{Mn})$ of the $80 \mathrm{~kg} / \mathrm{mol}$ ), was purchased from Aldrich, USA. Acetic acid and formic acid were obtained from Merck, Germany. nHAp (mean particle size of $<100 \mathrm{~nm}$ ) was received from Nanoshel, USA. Toluene and methylene chloride were distilled over calcium hydride. Other reagents were all of analytical grade and used as received without purification unless mentioned otherwise.

Culture medium of Dulbecco's Modified Eagle's medium (DMEM), fetal bovine serum, (FBS) penicillin-streptomycin, and trypsin-EDTA were all obtained from Gibco, UK. PBS (Merck, Germany), MTT (3-(4,5-dimethylthiazol-2-yl)-2,5-diphenyltetrazolium bromide) assay Kit (Sigma-Aldrich, UK), dimethyl sulfoxide (DMSO, Sigma-Aldrich, UK), 4,6diamino-2-phenylindole diacetate (DAPI, Sigma-Aldrich, UK), disposable hemacytometer(SKC Co., Ltd., Seoul, Korea), fluorescent-Inverted microscope (Bell, INV100FL, Japan), were also used in this study. Human fibroblast cells (HF), were purchased from Royan Stem Cell Technology Center of Iran (Tehran, Iran).

\subsection{Methods}

\subsubsection{Graft polymerization of $\varepsilon$-caprolactone chains on HAp nano-particles}

HAp ( $2 \mathrm{~g}$ ) was dried at $120^{\circ} \mathrm{C}$ under vacuum for $24 \mathrm{~h}$ and transferred to a flask containing $\varepsilon^{-}$ caprolactone ( $2 \mathrm{~g}, 0.175 \mathrm{mmol})$ and $\mathrm{Sn}(\mathrm{oct})_{2}(0.02 \mathrm{~g}$ in $20 \mathrm{ml}$ dry toluene), and stirred under a neat dry nitrogen purge. The reaction was maintained at $130^{\circ} \mathrm{C}$ for $7 \mathrm{~h}$. The mixture was cooled down to room temperature and diluted with methylene chloride. The PCL-g-HAp powder was separated by a repeated washing-centrifugation ( 3 cycles) at $2500 \mathrm{rpm}$ for 20 
min to remove the potentially unreacted $\varepsilon$-caprolactone and the free PCL homo-polymer chains formed during the reaction. Finally, the PCL-g-HAp particles were dried in a vacuum oven at room temperature for 24 hours.

\subsubsection{Preparation of PCL, PCL/HAp, PCL-g-HAp nano-fibrous scaffolds}

Nano-fibrous composite scaffolds consisting of the naked nHAp and the surface modified nHAp (PCL-g-HAp) nano-powders were developed through electrospinning technique. Poly ( $\varepsilon$-caprolactone) $(\mathrm{PCL}, \mathrm{MW}=80 \mathrm{~kg} / \mathrm{mol})$ solution was prepared at a concentration of $12.5 \%$ (w/v) in formic acid: acetic acid solvent system (6:4 vol ratio). PCL composites with nHAp or PCL-g-HAp (each at a fixed concentration of $5 \mathrm{wt} \%$ ), were prepared, separately. The prepared solutions were sonicated for $10 \mathrm{~min}$ and fed into a $2 \mathrm{ml}$ standard syringe equipped with a $0.7 \mathrm{~mm} \mathrm{G}$ blunted stain less steel needle using a syringe pump. The flow rate $(0.5$ $\mathrm{ml} / \mathrm{h})$, the distance between the needle and the collector $(18 \mathrm{~cm})$ and the voltage $(25 \mathrm{kv})$ were optimized and kept constant during the electrospinning process.

The scaffolds are named PCL, PCL/HAp and PCL/PCL-g-HAp, hereafter.

\section{Characterization}

\subsection{PCL-g-HAp characterization}

Spectroscopy: FTIR spectra were recorded on an Equinox 55 FTIR spectrometer in the range between 4000 and $400 \mathrm{~cm}^{-1}$. The HAp and PCL-g-HAp particles were mixed with $\mathrm{KBr}$ powder and pressed into discs prior to spectra acquisition.

Thermal Gravimetry: About 10-15 mg of HAp or PCL-g-HAp (as fine powder) were placed in a platinum sample pan of a TGA-PL 1500 (Polymer Laboratories, England). The heating rate was $10^{\circ} \mathrm{C} \cdot \mathrm{min}^{-1}$, and the samples were heated up to $650^{\circ} \mathrm{C}$.

Colloidal stability: The colloidal stability was measured using a separation analyzer (LUMiReader® 416.1; LUM, Germany). The specimens were prepared by dispersing nHAp 
or PCL-g-HAp nano-particles $(1 \mathrm{wt} \%)$ in chloroform followed by sonication for $2 \mathrm{~min}$. The colloidal stabilities were monitored for $12 \mathrm{~h}$ using a visible light at an intensity of $100 \%$.

\subsection{Scaffold Characterization}

\subsubsection{Morphological studies}

Morphologies of the electrospun nano-fibrous scaffolds were studied using a Tescan scanning electron microscope (vegaII X, Czech Republic), after gold coating. The fibers diameters of the electrospun mats were measured using the ImageJ software. Average fiber diameters and diameter distributions were calculated by the data obtained through averaging diameters of at least 100 random fibers in the SEM micrographs.

\subsubsection{Wettability; static water contact angle}

Contact angle measurements were performed on PCL and PCL/HAp, and PCL/PCL-g-HAp (5 wt\%) scaffolds by using a Kruss 40 apparatus at room temperature. Briefly, distilled water was dropped onto at least five different sites on each scaffold, and the static contact angle was measured. Results were reported as mean value + s.d.

\subsection{Biocompatibility studies; In vitro}

\subsubsection{In Vitro Bioactivity of the scaffolds}

In vitro bioactivity of the PCL/HAp and PCL/PCL-g-HAP nano-composite and control PCL nano-fibrous scaffolds were studied by incubating in Simulated Body Fluid (SBF). The SBF solution had a composition as reported in [20].

Scaffolds were cut as samples of $5 \mathrm{X} 5 \mathrm{~mm}^{2}$. Each specimen was immersed in $3 \mathrm{~mL}$ of SBF (about $15 \mathrm{mg} / 3 \mathrm{~mL}$ ) in screw capped polypropylene tubes. The bottles were placed in an orbital shaker at a constant speed of $60 \mathrm{rpm}$ and temperature of $37{ }^{\circ} \mathrm{C}$ for 15 days and the solution was refreshed every week. After incubation period, the samples were collected and 
rinsed thoroughly with phosphate-buffer saline (PBS) and ethanol and dried overnight at 37 ${ }^{\circ} \mathrm{C}$., then submitted for SEM and EDXA analysis.

\subsubsection{Human Fibroblast cells seeding on scaffolds}

HF cells in Dulbecco's Modified Eagle's Medium (DMEM), containing $10 \%$ fetal bovine serum (FBS), and $1 \%$ penicillin streptomycin, were grown in T-75 cell culture flask at $37^{\circ} \mathrm{C}$ in a humidified atmosphere of $5 \% \mathrm{CO}_{2}$. For sub-culturing, the cells, at $80-90 \%$ confluence, were washed in PBS, incubated in $1 \mathrm{ml}$ of $2.5 \%$ trypsin-EDTA at $37^{\circ} \mathrm{C}$ in a $\mathrm{CO}_{2}$ incubator, collected by centrifugation for $5 \mathrm{~min}$ and re-suspended in fresh DMEM. The cells were counted using a disposable hemocytometer under an inverted microscope, and the density was adjusted.

The prepared scaffolds $\left(1 \mathrm{~cm}^{2}\right)$, were sterilized with UV on both sides (20 min each), soaked in sterile PBS overnight and were seeded at a density of $10^{4}$ cells/scaffold and maintained in DMEM in an incubator at $37^{\circ} \mathrm{C}$ under $5 \% \mathrm{CO}_{2}$.

The PCL, PCL/HAp and PCL/PCL-g-HAp scaffolds were submitted then for cells-scaffolds interaction assessment using invert microscopy (Bell, INV-100FL, Japan), at definite time intervals.

\subsubsection{HF cells viability and proliferation}

To study the cells viability/proliferation on the prepared scaffolds, MTT (3-(4,5dimethylthiazol-2-yl)-2,5-diphenyltetrazolium bromide), assay was performed following the manufacturer's protocol. In brief, after 1, 2, 3, 7, 14 and 21 days of cells culture, $1 \mathrm{ml}$ of the MTT reagent as a mixture with DMEM (10 \%FBS) was added to each well of the culture plate containing the cultured scaffolds, and the plates were incubated for $4 \mathrm{~h}$ at $37^{\circ} \mathrm{C}$. After observing the formazan crystal formation, $200 \mu \mathrm{l}$ of DMSO was added to each well, to dissolving the formazan crystals. The plates were then incubated overnight. Next, 
measurement was carried out simultaneity at dual frequencies, 490 and $630 \mathrm{~nm}$ using an Elisa reader.

\subsubsection{HF cells attachment}

To observe the attached HF cells nuclei on the scaffolds after $24 \mathrm{~h}, 4$ ', 6'diamidino-2phenylindole diacetate (DAPI) staining was used. Cell/scaffold constructs were fixed in $4 \%$ formaldehyde in PBS for 10 min, washed twice with PBS, permeabilized with $0.1 \%$ Triton $\mathrm{X}-100$ in PBS for 20 min at $4^{\circ} \mathrm{C}$ and blocked with $1 \%$ Fetal Bovine Serum Albumin(FBSA) in PBS for 30 min at $37^{\circ} \mathrm{C}$. Nuclei and actin filaments in the cells were stained with DAPI, and were visualized using a fluorescent-inverted microscope (Bell, INV-100FL, Japan).

\subsubsection{HF Cells morphology}

To follow the cultured cell's morphology, cell/scaffold constructs were observed by SEM 48 and 72 hours after cell seeding. The scaffolds were washed with PBS twice, fixed with $4 \%$ glutaraldehyde for 3 hours, rinsed in PBS and dehydrated through a graded series of ethanol

$(30,40,50,60,70,80,90$ and $100 \%)$ at 10-min intervals. After air drying, the scaffolds were sputter coated with gold prior to SEM observation. The morphology of the attached cells on the scaffolds was also evaluated with confocal microscope.

\subsubsection{Adipose derived stem cells (ADMSCs) adhesion}

Prior to ADMSCs seeding, circular scaffolds were immersed in the following solutions: (1) $70 \%$ ethanol (1 h), (2) penicillin, streptomycin, and amphotericin B, and (3) culture medium to enhance cells attachment after seeding. Human adipose tissue derived stem cells (hADMSCs) were used for cell seeding. They were isolated from human adipose tissue, cultured and maintained in Dulbecco's modified Eagle's medium supplemented with $10 \%$ fetal bovine serum and a $1 \%$ antibiotic (10,000 U penicillin). hADMSCs were trypsinized and seeded on to the circular scaffolds withan initial cell density of $10^{4} \mathrm{~cm}^{-1}$ and incubated in the culture 
medium. Cells morphology on the scaffolds was evaluated by scanning electron microscopy. The cell-loaded scaffolds were rinsed with PBS after 3 days of cell seeding and fixed in glutaraldehyde $(2.5 \%)$ for $1 \mathrm{~h}$. For dehydration, the scaffolds were placed in a series of gradient alcohol concentration and then dried.

\subsubsection{Statistical analysis}

Statistical analysis of MTT results were performed using one-way analysis of variance (ANOVA). The data are presented as the mean \pm SD. When ANOVA indicated a significant difference among groups, the difference was evaluated using the least significant difference (LSD) and considered statistically significant at $\mathrm{p}<0.05$.

\section{Results and discussion}

\subsection{Characterization}

\subsubsection{Characterization of the PCL-g-HAp nano-particles}

Proper modification of HAp with graft polymerization of $\varepsilon$-caprolactone was confirmed using IR spectroscopy. Figure 1 shows the FTIR spectra of the pure PCL, HAp and the PCL-gHAp. The spectrum of HAp shows a band at $3565 \mathrm{~cm}^{-1}$ due to $\mathrm{OH}$ group of the HAp phase. The absorption peaks at 1028 and $962 \mathrm{~cm}^{-1}$ are attributed to stretching vibrations of the HAp phosphate group and peaks at 562 and $603 \mathrm{~cm}^{-1}$ are related to deformation vibration of the phosphate group. There are also $\mathrm{CO}_{3} v_{3}$ bands at $1420-1450 \mathrm{~cm}^{-1}$ and $v_{2}$ band at $864 \mathrm{~cm}^{-1}$ as an indication of carbonate-hydroxyapatite.

The wide absorption band at $3412 \mathrm{~cm}^{-1}$ and the absorption peaks at 1600 to $1650 \mathrm{~cm}^{-1}$ are assigned to presence of adsorbed $\mathrm{H}_{2} \mathrm{O}$ in the HAp. After surface grafting, a new absorption band appears at $1730 \mathrm{~cm}^{-1}$ belonging to the ester carbonyl group of PCL on the surface of 
PCL-g-HAp. New vibration band at $1575 \mathrm{~cm}^{-1}$ originated from the -OCO- vibration (Figure 1, left).

Colloidal stability of HAp nano-particles is important because of the particles tendency to aggregate in effect of van derWaals interactions as well as hydrogen bond formation between the surface hydroxyl groups. These interactions result in rapid precipitation in organic solution [10]. On the other hand, when nano-composites are made by solvent casting, a high colloidal stability is required to synthesize homogenous bulk nano-composites [10]. In other words, rapid precipitation of inorganic fillers in organic solvents cause inconsistent particle distribution within the polymer matrix. The LUMiReader dispersion analyses for the HAp and the PCL-g-HAp particles after 1 and 12 h, respectively, are shown in Figure 1, right. PCL-g-HAp featured a relatively high colloidal stability, while the HAp particles intensely precipitated after $1 \mathrm{~h}$. Phase separation developed within 10 min with HAp, as an indication of complete precipitation of the unmodified HAp in chloroform. Visual observation confirmed high colloidal stability of the PCL-g-HAp (not shown). Higher colloidal stability of the mixture containing PCL-g-HAp was attributed to surface modification of the HAp nano-particles. In fact, an amphiphilic structure was created by grafting PCL short chains onto the surface of HAp particles. The short PCL segments (hydrophobic part of the amphiphile), is soluble in chloroform and protects the HAp ends (hydrophilic part), from chloroform. The amphiphilic structure explains the reduced HAp interparticle interactions, which result in enhanced nano-particles stability in chloroform. Given the simple mixtures rule, PCL grafting also decreases the overall density of the HAp particles that favors the colloidal stability.

TGA experiments were carried out on both the as-received particles and the surface-modified apatite nano-particles to measure the amount of PCL chains grafted onto the nHAp surface (Figure 2A). HAp and PCL-g-HAp particles lost about $5.0 \%$ and $11.9 \%$ of weight on 
heating up to $650^{\circ} \mathrm{C}$, respectively. The amount of grafted PCL groups on the HAp surface was calculated as follows:

$\mathrm{M}_{\mathrm{PCL}}=\mathrm{WL}_{\mathrm{PCL}-\mathrm{g}-\mathrm{HAp}}-\mathrm{WL}_{\mathrm{HAp}}=11.9 \%-5.0 \%=6.9 \%$

Here, WLPCL-g-HAp denotes the weight loss of the PCL grafted nHAp particles and WLHAp is the weight loss of the as-received particles.

\section{Insert Figure 1}

\subsection{Morphology, wettability and mechanical properties of the scaffolds}

Optimization of the electrospinning parameters for fabrication of PCL, PCL/HAp and PCL/PCL-g-HAp fibers included systematic adjustment of the solution concentration, flow rate, working distance and voltage of the electrospinning condition to yield bead-free mats (Table 1). Based on literature reports, an optimal mass concentration of $12.5 \mathrm{w} / \mathrm{v} \% \mathrm{PCL}$ solution was selected for envisaged application of the bone tissue scaffolding [21-23]. In contrast to the smooth surface of the PCL nanofibers (Figure 2B), PCL/nHAp and PCL/PCLg-HAp nano-fibers contained nano-particles dispersed within the fibers (Figures 2C and D), which resulted in surfaces of higher roughness. However, the PCL/PCL-g-HAp nano-fibers exhibited smoother surface (Figures 2B-C) than the PCL/nHAp fibers, most possibly due to improved PCL/PCL-g-HAp interface. Also, a significant increase in the fiber diameter was registered for the PCL/nHAp and PCL/PCL-g-HAp nano-fibrous scaffolds compared to the neat PCL scaffold. Meanwhile, the mean fiber diameter decreased from PCL/nHAp to PCL/PCL-g-HAp where the nano-particle- polymer interface was modified by taking a surface modification approach (Table 2).

\section{Insert Figure 2}


Hydrophilicity of both the manufactured nano-composite scaffolds were examined by measuring the water contact angle and the results were compared with those recorded for the neat PCL (Table 2).

The water contact angles (WCA) of PCL/HAp and PCL/PCL-g-HAp nano-composite scaffolds are lower than that of the neat PCL, indicating that dispersion of HAp and PCL-gHAp nano-particles in the polymeric matrix makes the surface more hydrophilic. However, slightly larger WCA was measured for the PCL/PCL-g-HAp nano-composite scaffold as an indication of the improved polymer/nano-particles interfacial interaction.

All of these observations might be ascribed to the synergetic contribution of both surface chemistry and topography that can be clearly varied by including the HAp and PCL-g-HAp into the nano-composite scaffolds as discussed earlier. This may clarify the remarkable observed differences between the WCA values recorded for the sample prepared as film or scaffold. Our results (Table 2), are in close agreement with the values reported for PCL and PCL-nano-composite scaffolds in the literature.[24]

The representative tensile stress-strain curves of PCL, PCL/HAp and PCL/PCL-g-HAp scaffolds are shown in Figure 3. The results show that HAp and PCL-g-HAp significantly improved the mechanical properties of the scaffolds.

Composite scaffolds consisting of polymers reinforced with ceramic nano-particles are widely applied for hard tissue engineering. However, due to the incompatible polarities of the ceramic nano-particles with polymers, they tend to agglomerate in the polymer matrix which results in undesirable effects on the integral properties of composites.

According to mechanical properties results, surface modification of HAp with $\varepsilon$-caprolactone increased the tensile strength and modulus of the PCL/PCL-g-HAp nano-composite scaffold 
up to about 10 and 33 percent compared to those of the PCL/HAp, respectively (Figure 3). This is due to improved compatibility between the matrix and the filler and enhanced adhesion at the organic-inorganic inter-phase as well as the uniform dispersion of HAp at nano-scale within the polymer matrix. Similar mechanical behavior was previously reported by Shokrollahi et al., when tried to improve HAp/PCL inter-phase in a PCL/HAp supramolecular nano-composite [10].

\section{Insert Figure 3}

\subsection{Apatite formation on the scaffolds}

SEM micrographs of the PCL/HAp and PCL/PCL-g-HAp nano-composites and PCL nanofibrous scaffolds incubated in SBF for 15 days are shown in Figure 4. In obvious contrast to the as prepared PCL/HAp and PCL/PCL-g-HAp scaffolds(Figure 2), were covered with a relatively thick layer of crystallites apatite. The observed morphology of the HAp layer on the surface of both the scaffolds was similar. To the contrary, no apatite layer deposition was observed on the PCL control (Figure 2), at the same time point.

To demonstrate the composition of the inorganic layer on the specimens surfaces, EDX elemental analysis was performed on PCL/HAp and PCL/PCL-g-HAp nano-composite scaffolds (Figure 4). Calcium phosphate nature of the deposits on both the composite samples was shown by EDXA analysis and the results demonstrated a $\mathrm{Ca} / \mathrm{P}$ ratio of 1.60 and 1.72 for PCL/HAp and PCL/PCL-g-HAp nano-composites, respectively. These results indicate that the inorganic layer formed on the nano-biocomposite scaffolds is hydroxyapatite. Recently, Allo et al., designed a set of experiments to evaluate bioactivity of PCL/bioglass composites by monitoring HAp deposition on the samples upon incubation in SBF. They have shown that both the incubation period and the bioglass concentration could control amount of the HAP deposition.[25] In the present study, there is much similarity 
between morphologies of the apatite layer on PCL/HAp and PCL/PCL-g-HAp nanocomposites. Therefore, it can be concluded that PCL grafting on the surface of the HAp nanoparticles did not result in any reduction in the bioactivity of the PCL/PCL-g-HAp scaffold compared to the PCL/HAp scaffold.

\subsection{Viability and proliferation of fibroblasts}

After 24 hours of cell seeding, Human Fibroblasts were observed by using of inverted microscope (Bell, INV-100FL, Japan). Images showed that all scaffolds are biocompatible against HF cells, because as it could be seen in Figure 5, HFs adhered to the scaffold and show normal structure of Human Fibroblasts morphology, beside the scaffolds.

\section{Insert Figure 5}

The results of MTT assay after cell seeding on the scaffolds are shown in Figure 6. As it is illustrated in Figure 6, the HF cells on the bioceramic/PCL nano-composites (both the PCL/HAp and the PCL/PCL-g-HAp), have shown more proliferation compared to the PCL by the end of day 7 . After 14 days, the control showed more proliferation in comparison with the PCL. Meanwhile, the PCL/PCL-g-HAp nano-composite stood ahead of PCL, PCL/HAp and the control in terms of the cells proliferation. When the experiment prolonged until 21 days, this difference became even more pronounced $(\mathrm{P}<0.05)$.

\section{Insert Figure 6}

Influence of HAp on bone cells and osteoblastic differentiation of MSCs was studied by different research groups. For example, Wutticharoenmongkol et al., have studied MC3T3-E1 cells behavior on two types of PCL and PCL-HAp scaffolds prepared by electrospinning. 
They concluded that electrospun PCL/HAp scaffold showed good bone regeneration evidenced by a high degree of osteocalcin expression on day 21 [26].

Lao et al., have reported on uptrend cell proliferation of MC3T3-E1 cells on a PLGA-HAp scaffold within 7 days after culture, which was followed by a decrease in the cells number after 14 days. The alkaline phosphatase activity of cells was higher on PLGA-HAp scaffold compared to those on the PLGA scaffold and the control [27].

Fernandez et al., have investigated two osteoblast cell lines (UMR 106 and MC3T3E1) behavior on three types of scaffolds; HAp, PCL and a hybrid poly-D-isopropyl fumarate/polycaprolactone/hydroxyapatite scaffold (PDIPE-PCL-HAp). They have reported remarkably higher mechanical properties for the hybrid scaffold compared even to those of the pure HAp. Secretion of collagen type 1 was also high with both the cell lines on the hybrid scaffold. Therefore, they concluded that HAp stimulated production of collagen type 1, and simultaneously improved mechanical properties of the scaffolds [28].

Nano-HAp plays dual roles of improving the mechanical properties of the nano-composites and providing a favorable environment for osteoblasts adhesion and proliferation [8,9]. A good interfacial adhesion between organic and inorganic phases, namely the polymer matrix and the HAp particles, and uniform dispersion of HAp at nano-level in the polymer matrix are the two crucial parameters that control versatility of the resulting nano-composites in tissue engineering applications [18]. Mechanical properties and cell adhesion/proliferation properties of nano-composites are greatly influenced by the organic/inorganic interface and the HAp dispersion, respectively. From a biological view point, HA dispersion at nano scale is very important simply because it provides a larger surface available for the cultured cells. Kim et al., have demonstrated that HAp surface-modified with PCL (with a graft content of about $24 \mathrm{wt} \%$ ) contributed to the improved mechanical strength of PCL/HAp nano- 
composites (about 20\% enhancement in tensile strength as compared to the conventional PCL/HAp nano-composite), due to the enhanced adhesion of PCL and HAp at interface especially because PCL-grafted HAp nano-crystals were used for composite preparation [18]. However, when mouse fibroblasts were cultured on the PCL/PCL grafted HAp nanocomposites in their study significant enhancement of the cells proliferation(compared to the neat PCL), was only observed for the composites containing $30 \mathrm{wt} \%$ of the surface modified bio-ceramic 7 days after cells seeding [17]. On the contrary, in our study addition of 5wt $\%$ PCL-g-HAp nano-particles (with a graft content of about $7 \mathrm{wt} \%$ ), to PCL resulted in remarkable increase in the fibroblasts proliferation (compared to the neat $\mathrm{PCL}, \mathrm{P}<0.01$ ), 7 days post culture (Figure 6). This comparison illuminates that there is an optimum graft density at which both the mechanical properties and the bioactivity improve remarkably.

Next, we extended our cell studies to staining the cells nuclei with 4', 6'-diamino-2phenylindole diacetate (DAPI) reagent. Based on DAPI staining results (Figure 7), after $24 \mathrm{~h}$, the PCL/PCL-g-HAp bio-nano-composite scaffold showed more affinity to adhesion and attachment of HFs. DAPI images clearly show that PCL/PCL-g-HAp scaffold provided a more uniform attachment of human fibroblasts as evidenced by DAPI-stained cell nuclei (Figure 7).

\section{Insert Figure 7}

To provide a better understanding of cell-scaffold interaction, SEM observation was carried out at definite time period of 48 hours after cell seeding (Figure 8). As is clearly seen in SEM micrographs, PCL/PCL-g-HAp nano-composite scaffold enjoys a better cell connectivity and cell morphology than other scaffolds (Figure 8). A close observation of the pure PCL scaffold reveals that no cells are accommodated at some area, while cell aggregates are formed at some other areas. After 21 days, surface of the PCL/PCL-g-HAp scaffold was 
covered by a cell sheet that might be skin cell layers. These observations show that grafting of HAp with short PCL chains could result in nano-composites of superior mechanical properties with enhanced ability to cell localizations and normal signaling. This scaffold also resulted in uniform cell distribution, as an indication of improved cell-cell signaling (Figures 5c, 7c, and 8).

\section{Insert Figure 8}

Cells in general and fibroblasts, in particular, are matrix dependent; they attach the surface when seeded onto the scaffolds with appropriate physico-chemical properties [26, 29]. Therefore, cell adhesion happens after cell seeding, and cells initiate recognizing the surrounding micro-environment, sending peripheral signaling. Cells then provide cellular connections with expanding actin fibers and express integrin receptors [28]. Given it must encourage natural cell proliferation by providing mechanical support until the new tissue is finally remodeled, a key requirement for successful function of any tissue engineering scaffold is the mechanical properties [30]. In this study, it was observed that the PCL/PCL-gHAp nano-composite scaffold improved the cells proliferation and cell-cell connections until the end of day 21 of culture as according to MTT assay results as well as cell morphology studies (Figure 9). A remarkable increase in integrin binding sites is prerequisite for such event [31]. Integrin transmembrane proteins act similar to other protein receptors (proteins that play a role in cell-cell signaling and at the meanwhile integrins are known as focal points of the cell cytoskeleton. Cell cytoskeleton is completely depended on calcium ions. Most of intermediate filaments including vimentin, laminin and keratin need $\mathrm{Ca}^{+2}$ for their performance [32]. Integrin proteins in cell membrane are involved in interaction with cytoskeleton components and also use many pathways to cell-peripheral micro-environment messaging to transferring data from the cell to the ECM as well as to the adjacent cells. Integrin amino acid sequence is cysteine-rich with binding sites for divalent cations such as 
$\mathrm{Ca}^{+2}$. According to our cell study results the cells preferably localized themselves onto the PCL/PCL-g-HAp nano-composite scaffold when cell culture was continued until day 21 . Based on the observed cell morphology and the MTT results it seems that the PCL/PCL-gHAp nano-composite scaffold could absorb $\mathrm{Ca}^{+2}$ receptor proteins from the membrane of fibroblast cells at a higher rate than either the PCL or the PCL/HAp scaffolds[32].

\section{Insert Figure 9}

It can be concluded therefore that surface grafting of HAp nano-particles with PCL short chains improves PCL/bio-ceramic compatibility at interphase and hence, the mechanical properties of PCL/PCL-g-HAp enhanced remarkably. Mechanical properties enhancement in polymer/filler nano-composites is attributed to uniform distribution of finely dispersed (most possibly at nano-level), nano-particles within the polymer matrix, which is in perfect agreement with a previous report by Shokrollahi et al [11]. An immediate result of such fine dispersion of bioceramic nano-particles within the PCL matrix would be more bioceramic sites (as a source of $\mathrm{Ca}^{2+}$ ions), available to the cultured cells, particularly in comparison with PCL/HAp nano-composite scaffold, where bioceramics particle agglomeration happened and resulted in relatively weaker mechanical properties [23].

This conclusion is clearly evidenced by results from MTT assay, DAPI staining and SEM observation. We conclude therefore that, even short time ( 7 hours versus more than 15 hours as was previously reported by Lee et al.,[18] grafting of PCL chains on HAp nano-particles resulted in PCL/PCL-g-HAp electrospun nano-composite scaffold with enhanced $\mathrm{Ca}^{+2}$ dependent cell attachment. We believe that the observed enhancement in cells attachment is a direct result of improved dispersability of HAp nano-particles within the PCL matrix, reflected in better cells morphology and suitable localization, which triggers adequate cellcell signaling. Perfect cell signaling results in more and correct proliferation in tissue regeneration. 


\subsection{Adhesion of adipose derived stem cells (ADMSCs)}

Considering the final application of the scaffolds as bone tissue engineering material, adhesion of adipose derived stem cells (ADMSCs) on the PCL, the PCL/HAp, and the PCL/PCL-g-HAp electrospun nano-composite scaffolds was evaluated next. The ADMSCs behavior on scaffolds is displayed in Figure 10 As it can be seen in the Figure 10, almost a cell sheet was formed on the PCL/PCL-g-HAp. In contrast to the naked PCL scaffold, the cells also covered large areas on the surface of PCL/HAp scaffold. Based on these preliminary cell experiments, it can be concluded that the PCL/PCL-g-HAp scaffold, provides more cell binding sites than the PCL and the /PCL/HAp scaffolds.

Insert Figure 10

\section{Conclusion}

Relatively short chains of PCL were grafted on the surface of HAp nano-particles through ring opening polymerization of $\varepsilon$-caprolactone (PCL-g-HAp). FTIR and TGA studies results showed that PCL (6.9 wt\%), was successfully grafted on the HAp resulting in nano-particles of relatively high colloidal stability(PCL-g-HAp). PCL, PCL/HAp, and PCL/PCL-g-HAp scaffolds were prepared by electrospinning. PCL/PCL-g-HAp nano-composite scaffold showed about 10 and 33 percent increase in tensile strength and modulus, respectively, compared to those of the PCL/HAp.

HAp formation on the nano-composite scaffolds after 15 days incubation in simulated body fluid (SBF), showed no significant difference between the PCL/HAp, and PCL/PCL-g-HAp scaffolds, indicating that bioactivity of PCL/PCL-g-HAp scaffold remained intact upon grafting. Human fibroblasts were seeded on all the scaffolds. According to MTT assay results, the highest ability in induction of the cells proliferation was registered for PCL/PCLg-HAp nano-composite. Cells were stained with DAPI and fluorescent microscopy of the 
DAPI stained samples and electron microscopy images showed that all the nano-fibrous scaffolds (PCL, PCL/HAp, and PCL/PCL-g-HAp), were non-toxic against cells, while more cell adhesion, and the most uniform cell distribution observed on PCL/PCL-g-HAp. According to the results obtained, we suggest that grafting of relatively short chains of PCL on the surface of HAp nano-particles stimulates fibroblasts/ADMSCs adhesion and fibroblasts proliferation on the PCL/PCL-g-nHAp nano-composite.

\section{Acknowledgment}

This work was supported by Iran National Science Foundation (INSF), grant No.89000913.

\section{References}

1. Park J, Kim S, Moon S, Kim C, Yoon H. Establishment and maintenance of human embryonic stem cells on STO, a permanently growing cell line. Biol Reprod. 2003; 69: 20072014.

2. Hutmacher D.W., State of the art and future directions of scaffold- based bone engineering from a biomaterials perspective. Tissue Eng Regen Med. 2007; 1:245-60.

3. Ma Z, Kotaki M, Inai R, Ramakrishna S. Potential of nano-fiber matrix as tissueengineering scaffolds. Tissue Eng. 2005; 11:101-9. 
4. Liu, Z., et al. Filling behavior, morphology evolution and crystallization behavior of microinjection molded poly(lactic acid)/hydroxyapatite nanocomposites. Compos Part A: Appl Sci. 2015;72: 85-95.

5. Wong, S-C, Baji A, and Gent AN. Effect of specimen thickness on fracture toughness and adhesive properties of hydroxyapatite-filled polycaprolactone. Compos Part A: Appl Sci. 2008;39:579-587.

6. Sahay R, Suresh Kumar P, Sridhar R, Sundaramurthy J, Venugopal J, Mhaisalkar S, Ramakrishna S. Electrospun composite nanofibers and their multifaced applications. Mater Chem. 2012; 22:12953-12971

7. Akihito $\mathrm{K}$, et al. Combination of root surface modification with BMP-2 and collagen hydrogel scaffold implantation for periodontal healing in beagle dogs. Open Dent J. 2015; 9: $52-59$

8. Liu X, Won Y and X.Ma P. Porogen-induced surface modification of nano-fibrous poly (L-lactic acid) scaffolds for tissue engineering. Biomaterials. 2006; 27:3980-3987.

9. Yang X.B, et al. Human osteoprogenitor growth and differentiation on synthetic biodegradable structures after surface modification. Bone. 2001; 29:523-531

10. Mehmanchi M, Shokrollahi P, Supramolecular polycaprolactone nanocomposite based on functionalized hydroxyapatite. J Bioact Compat Polym. 2012; 27:467-480

11. Shokrollahi P, Mehmanchi M, et al. Effect of interface on mechanical properties and biodegradation of PCL HAp supramolecular nano-composites. Mater Sci Mater Med. 2014; 25:23-35

12. Hong Z, Qiu X, Sun J, et al. Grafting polymerization of l-lactide on the surface of hydroxyapatite nano-crystals. Polymer. 2004; 45:6699-6706

13. Liu F, Wang R, et al. Polymer grafted hydroxyapatite whisker as a filler for dental composite resin with en

14. hanced physical and mechanical properties. Mater Sci Eng C Mater Biol Appl. 2013; 33:4994-5000

15. Qiu X, Hong Z, Hu J, et al. Hydroxyapatite Surface Modified by l-Lactic Acid and Its Subsequent Grafting Polymerization of 1-Lactide, Biomacromolecules. 2005; 6:1193-1199

16. Shan Y, Qin Y, et al. The Synthesis and Characterization of Hydroxyapatite- $\beta$ Alanine Modified by Grafting Polymerization of $\gamma$-Benzyl-L-glutamate-N-carboxyanhydride, Molecules. 2013; 18:13979-13991

17. Zeng L.Wng H, et al. A new approach for synthesis of the comb-shaped poly (ecaprolactone) brushes on the surface of nano-hydroxyapatite by combination of ATRP and ROP. Coll Interf Sci. 2010; 352:36-42

18. Lee HJ, Choi HW, et al. Modification of Hydroxyapatite Nanosurfaces for Enhanced Colloidal Stability and Improved Interfacial Adhesion in Nanocomposites. Chem Mater. 2006; 21:5111-5118

19. Lee HJ, Kim SE, et al. The effect of surface-modified nano-hydroxyapatite on biocompatibility of poly(e-caprolactone)/hydroxyapatite nanocomposites. Eur Polym J. 2007; 43:1602-1608

20. Kokubo T, Kushitani H, et al., Solutions able to reproduce in vivo surface-structure changes in bioactive glass-ceramic A-W. Biomed Mater Res. 1990; 24:721-734. 
21. Shokrollahi P, Mirzadeh H, Scherman OA, Huck WTS. Biological and mechanical properties of novel composites based on supramolecular polycaprolactone and functionalized hydroxyapatite. Biomed Mater Res A. 2010; 95:209-221

22. Van der Schueren L, De Schoenmaker B. An alternative solvent system for the steady state electrospinning of polycaprolactone. Eur Polym J. 2011; 47:1256-1263

23. Ghorbani FM, Kaffashi B, Shokrollahi P, et al. PCL/chitosan/Zn-doped nHA electrospun nanocomposite scaffoldpromotes adipose derived stem cells adhesion and proliferation. Carbohydr Polym. 2015; 118:133-142

24. Park S, Lee S, Kim W. Fabrication of porous polycaprolactone/hydroxyapatite (PCL/HA) blend scaffolds using a 3D plotting system for bone tissue engineering. Bioproc Biosys Eng. 2011;34:505-513

25. Allo BA, Rizkalla AS, Mequanint K, Hydroxyapatite Formation on Sol-Gel Derived Poly( $\varepsilon$-Caprolactone)/Bioactive Glass Hybrid Biomaterials, ACS Appl. Mater. Interfaces 2012; 4: 3148-3156

26. Chih-Hao C, Ming-Yih L, et al., Surface modification of polycaprolactone scaffolds fabricated via selective laser sintering for cartilage tissue engineering. Mater Sci Eng C. 2014;40:389-397

27. Wutticharoenmongkol P, Pavasant P, Supaphol P .Osteoblastic Phenotype Expression of MC3T3-E1 Cultured on Electrospun Polycaprolactone Fiber Mats Filled with Hydroxyapatite Nanoparticles. Biomacromolecules. 2007;8:2602-2610

28. Lao L, Wang Y, Zhu Y, Zhang Y, Gao Ch. Poly(lactide-co-glycolide)/hydroxyapatite nanofiberous scaffolds fabricated by electrospinning for bone tissue engineering. J Mater Sci Mater Med. 2011; 22:1873-1884

29. Fernandez J, Molinuevo M. Development of an osteoconductive PCL-PDIPFhydroxyapatite composite scaffold for bone tissue engineering. Tissue Eng Regen Med. 2011; 6:126-135

30. Sharma S, Pawar A. Low density multiparticulate system for pulsatile release of meloxicam. Internat J Pharm. 2006; 313:150-8.

31. Ruckh T, Carroll D, Weaver J, Popat K. Mineralization Content Alters Osteogenic Responses of Bone Marrow Stromal Cells on Hydroxyapatite/Polycaprolactone Composite Nanofiber Scaffolds. Funct Biomater. 2012; 3:776-798

32. Martinez T, Gomez J, Ribelles D, Ferrer G., Fibrin coating on poly (L-lactide) scaffolds for tissue engineering. J Bioact compat polym. 2011; 26: 464-474 


\section{Figures captions}

Figure 1. Left: FTIR spectra of PCL, HAp and PCL-g-HAp; Right: comparative colloidal stability of the naked HAp nano-particle (up, $1 \mathrm{~h}$ after preparation) and PCL-g-HAp (down, $12 \mathrm{~h}$ after preparation).

Figure 2. TGA curves of the HAp and the PCL-g-HAp nano-particles (A). SEM micrographs of C: PCL, E: PCL/HAp, and G: PCL/PCL-g-HAp nano-fibers prepared in HF/HA mixed solvent system applying the spinning conditions as indicated in Table 1. A higher magnification of the figures B, D, and F are shown in Figures C, E and G. The PCL/HAp nano-fibers exhibited surface of higher roughness compared to the neat PCL and the PCL/PCL-g-HAp (arrows on C inset).

Figure 3. Representative stress-strain curves as well as variation in tensile modulus, tensile strength and tensile strain of the PCL, PCL/HAp and PCL-g-HAp nano-fibrous scaffolds.

Figure 4. SEM micrographs showing the evolution of the deposition of HAp layer on PCL/HAp and PCL/PCL-g-HAp composite scaffolds after 15 days incubation in SBF.

Figure 5. Optical microscope photographs showing Human Fibroblasts cells behavior on a: PCL, b: PCL/ HAp and c: PCL/PCL-g-HAp scaffolds 24 hours after cell seeding. Black pointer shows region of scaffolds in cell culture medium.

Figure 6. Viability and proliferation of human fibroblasts on the PCL, PCL/HAp, and PCL/PCL-g-HAp nano-composite scaffolds as a function of culture time $(\mathrm{a}: \mathrm{P}<0.05$, $\mathrm{b}: \mathrm{P}<0.01, \mathrm{c}: \mathrm{P}<0.001)$. 
Figure 7 DAPI staining of the human fibroblast cells nuclie on; a: PCL, b: PCL/ HAp and c: PCL/PCL-g-HAp scaffolds.

Figure 8. SEM images show cell morphology on PCL, PCL/HAp, and PCL/PCL-g-HAp nano-composite scaffolds after culture for 48 hours. HF cells were seeded at a density of $10^{4}$ cells/well.

Figure 9. SEM images show cell morphology on PCL-g-HAp nano-composite scaffold after 3, 7 and 14 days of culture shows progressive increase in cells density. HF cells were seeded at a density of $10^{4}$ cells/well.

Figure 10. SEM images show ADMSCs morphology on PCL, PCL/HAp, and PCL/PCL-gHAp nano-composite scaffolds after culture for 5 days.

\section{Table caption}

Table 1. Electrospinning optimized process parameters and fiber diameters of PCL, PCL/HAp, and PCL/PCL-g-HAp nanofibrous scaffolds. RH of 30 $\pm 4 \%$, Solvent: HF/HA (60:40 V/V\%), and PCL concentration 12.5 wt \% keep constant.

Table 2. Water contact angels of PCL, PCL/HAp, and PCL/PCL-g-HAp as films and nanofibrous scaffolds. 

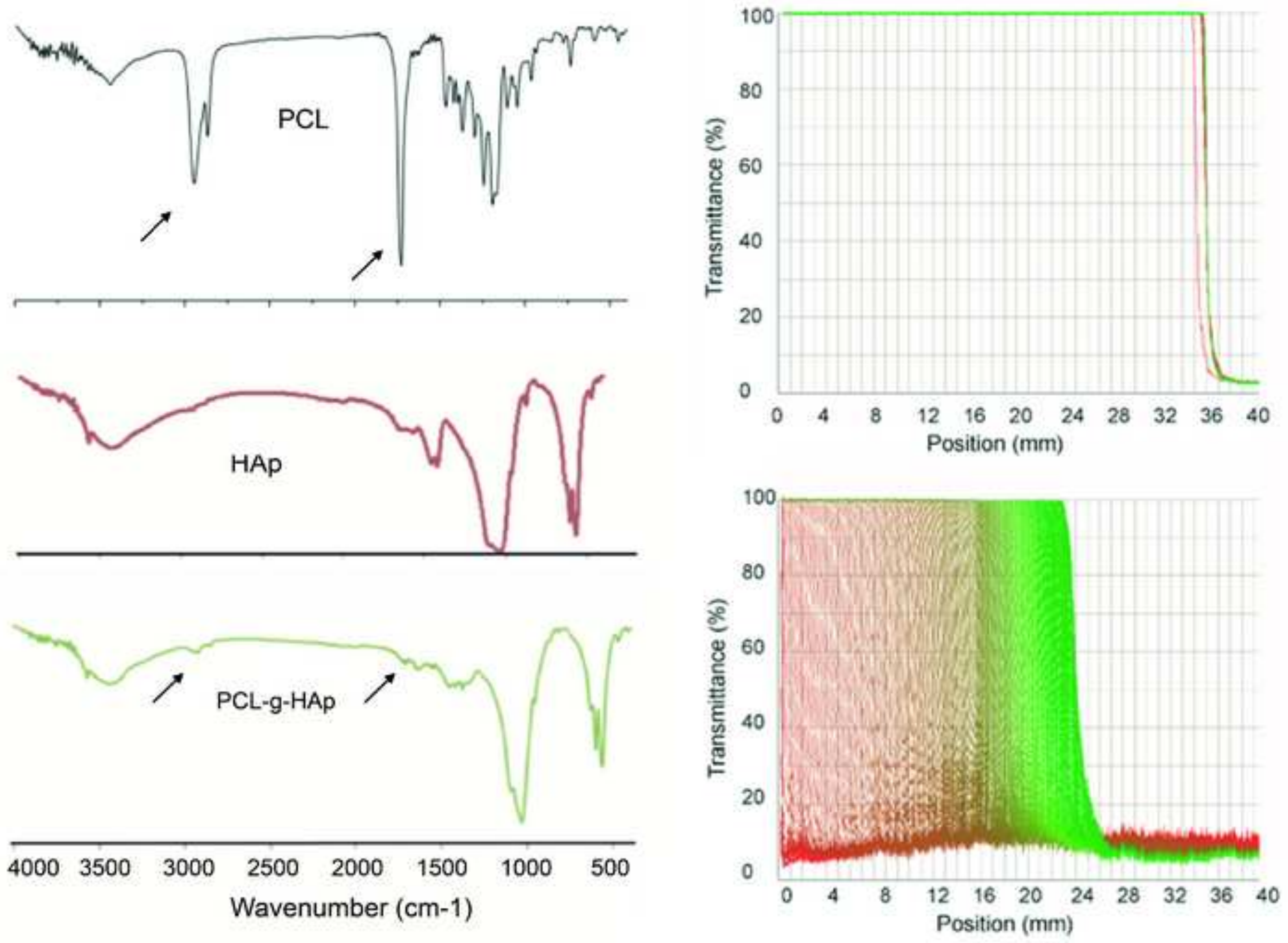

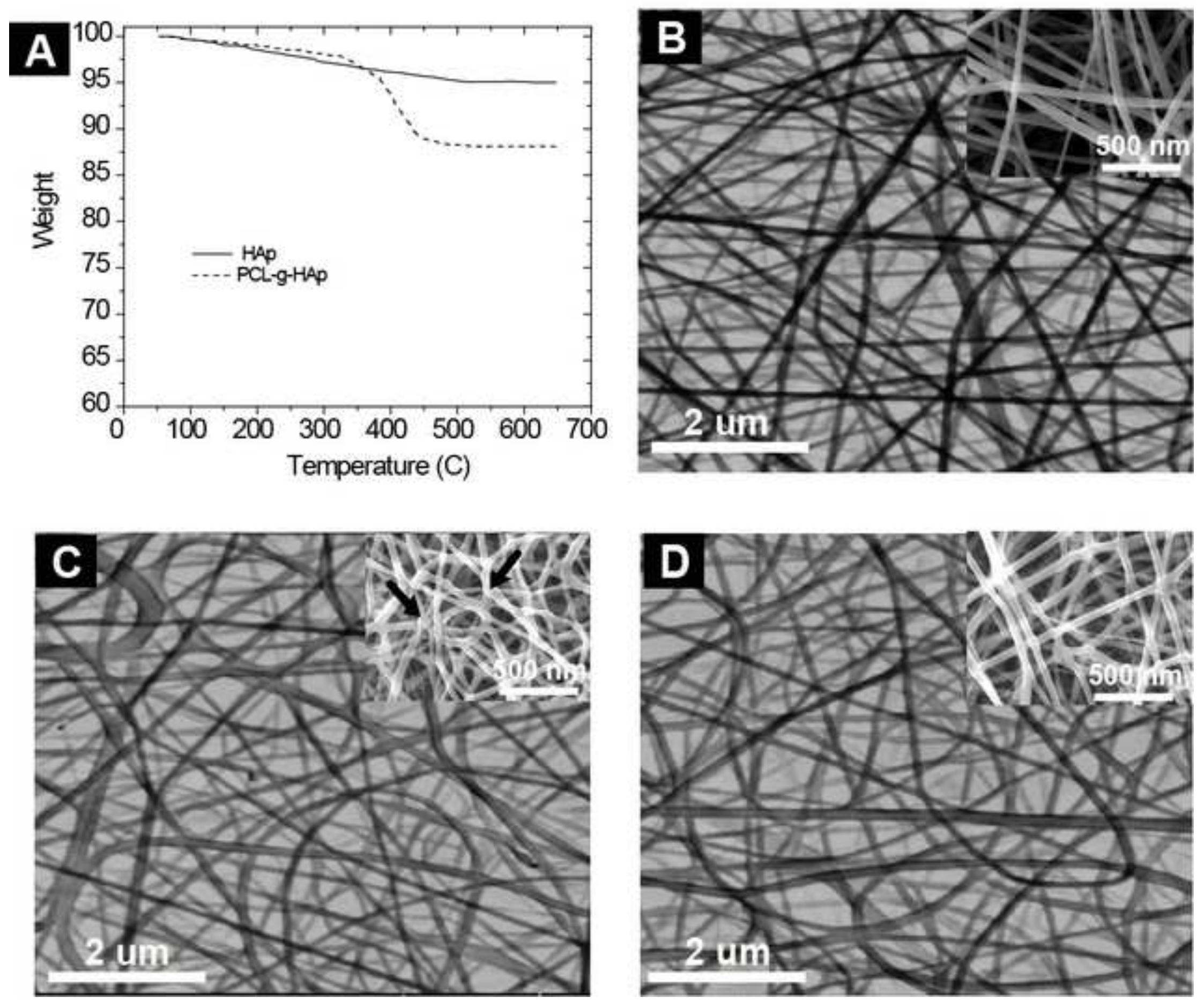

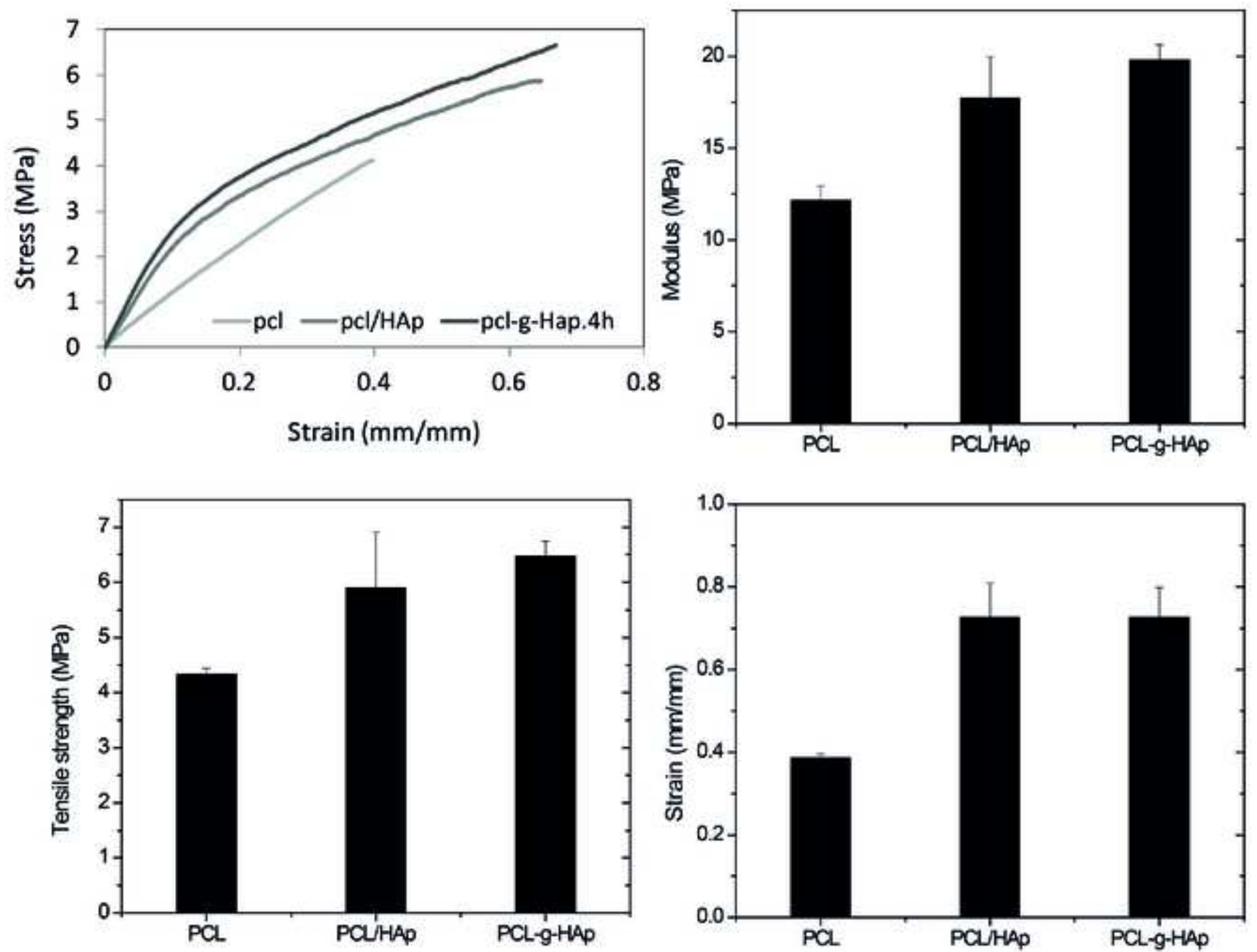

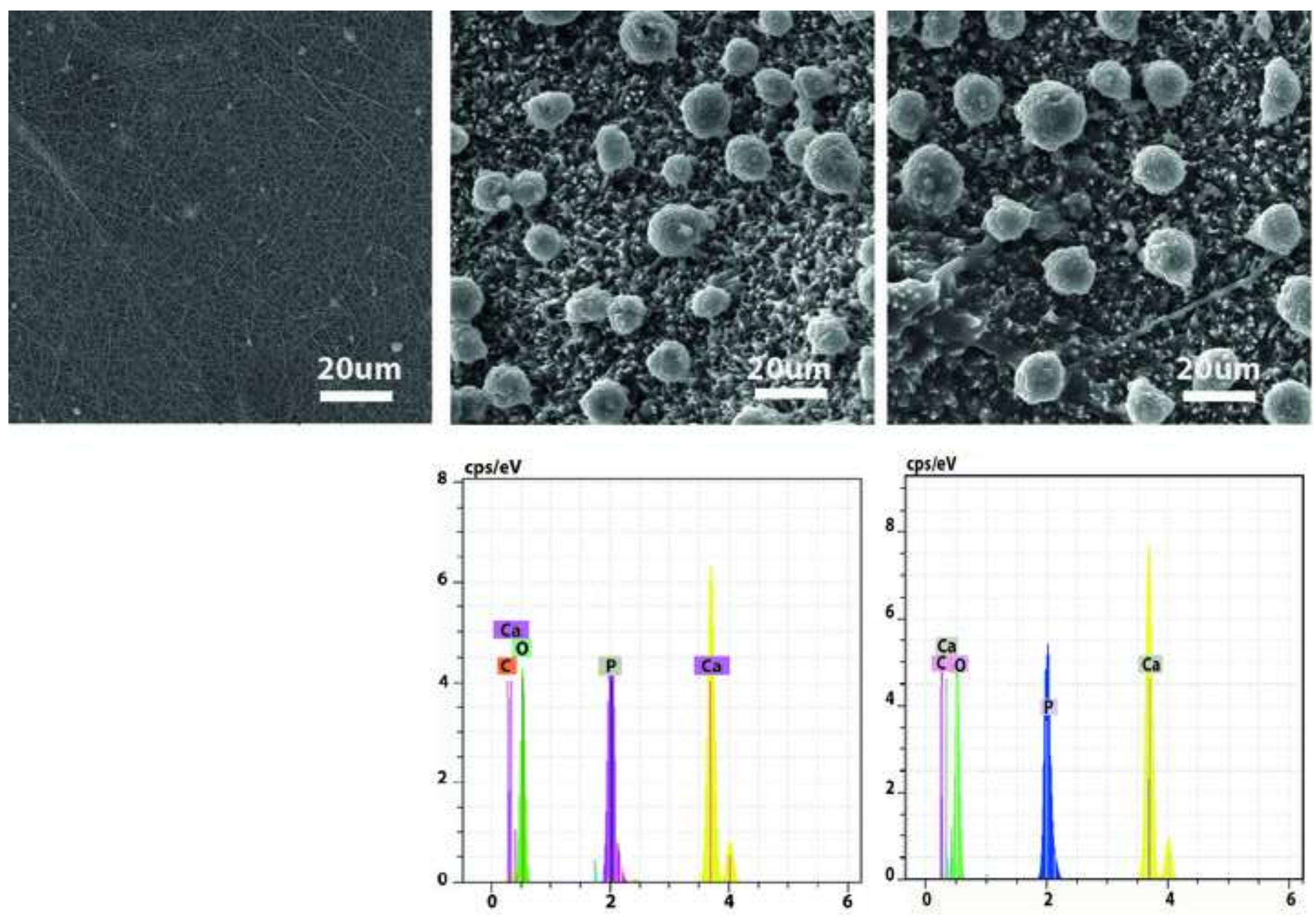


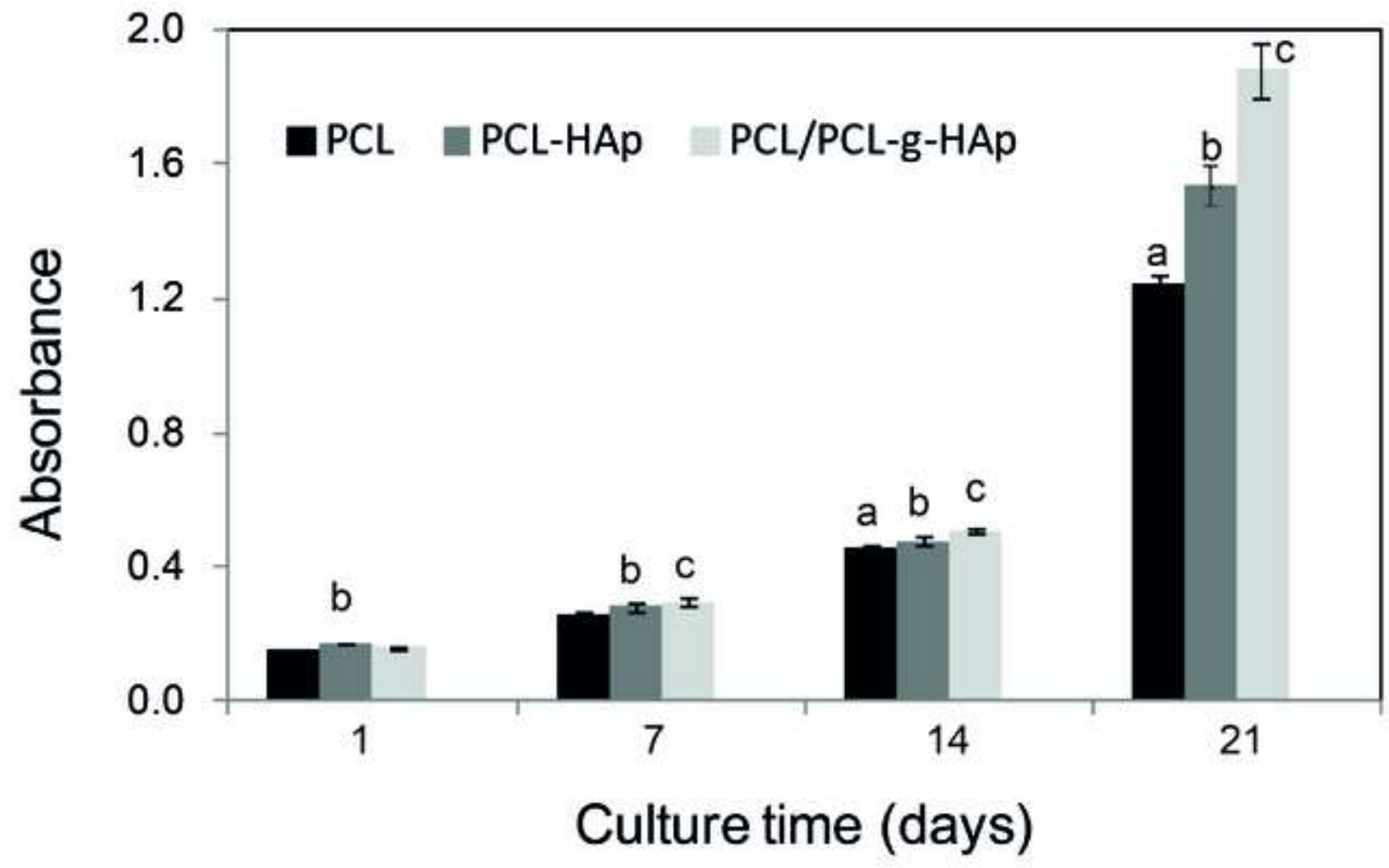




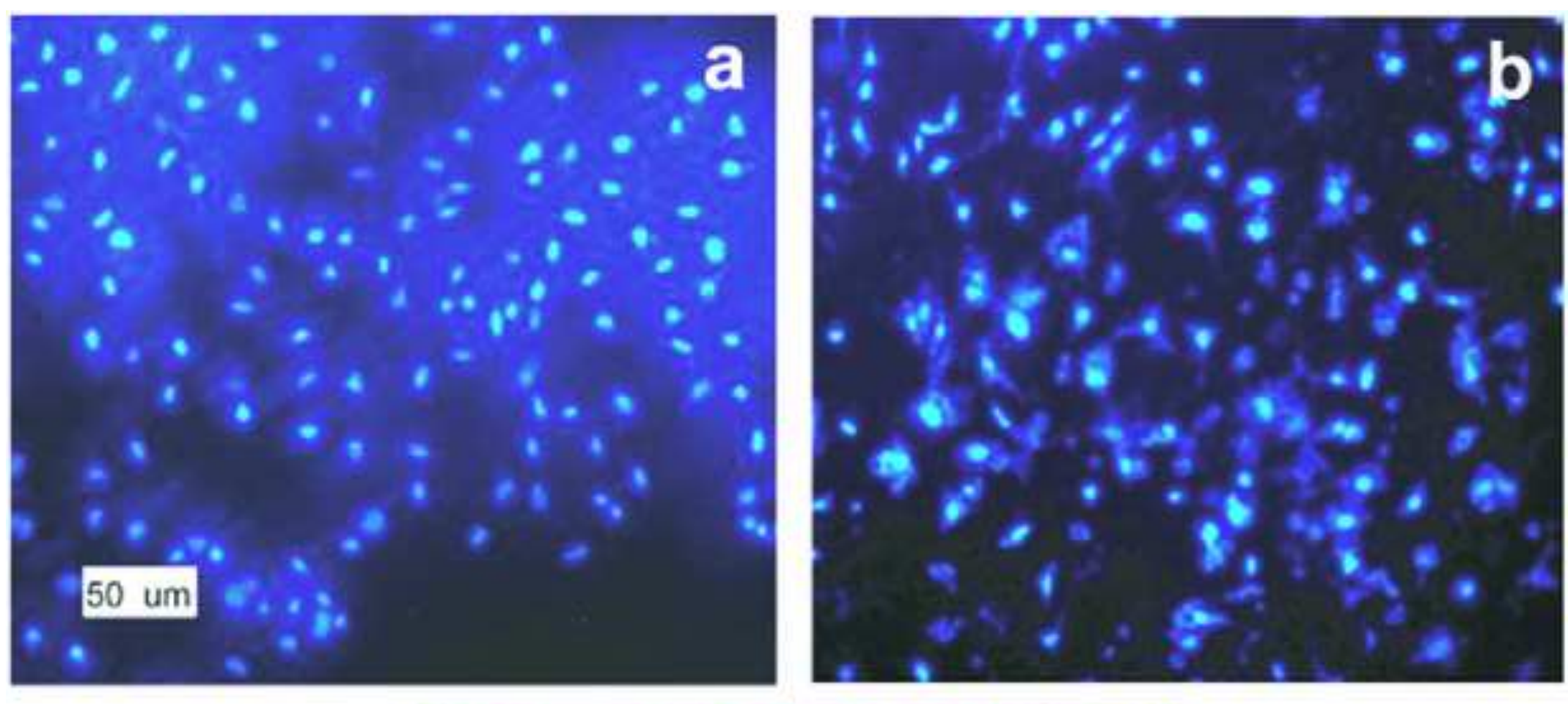

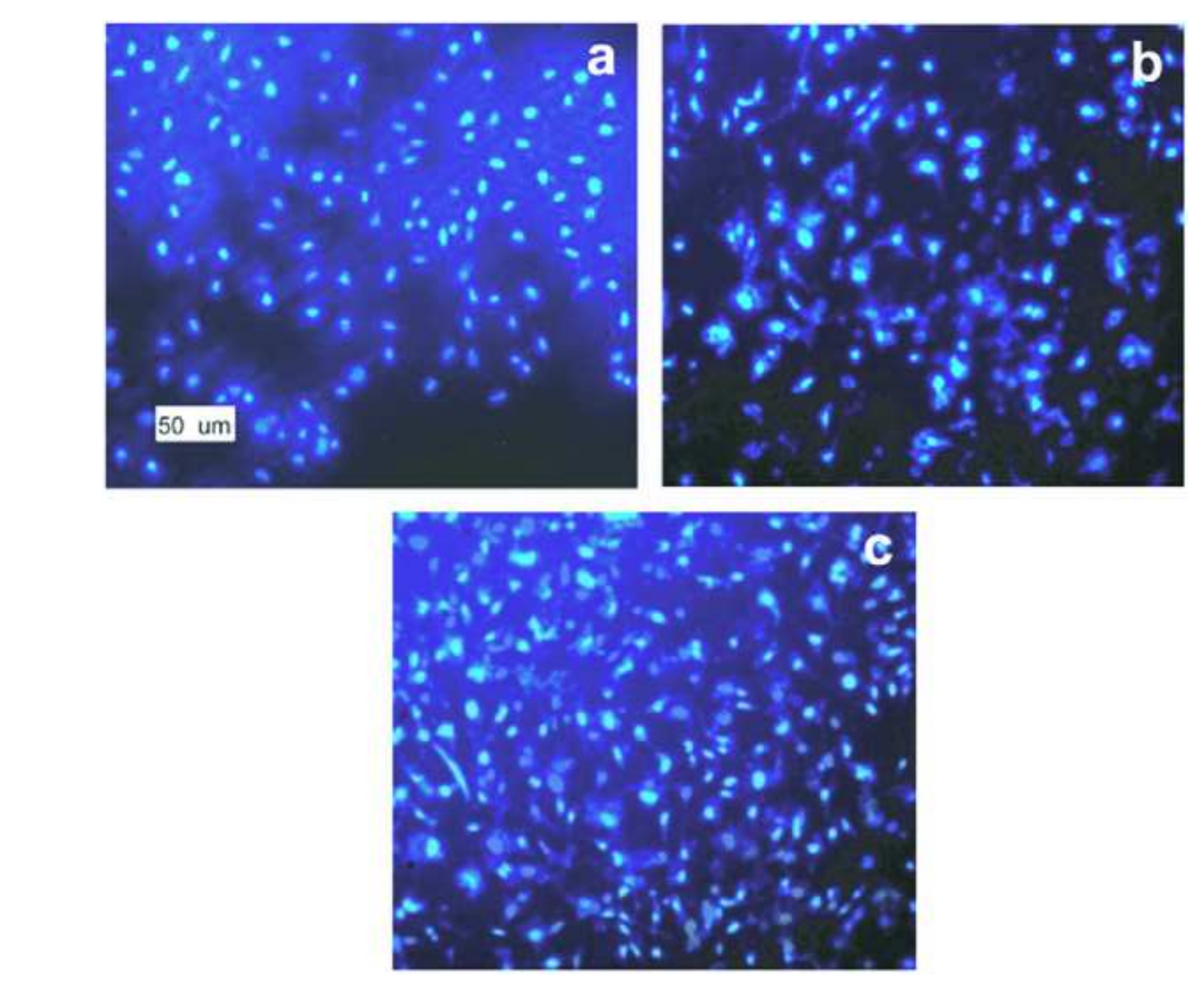

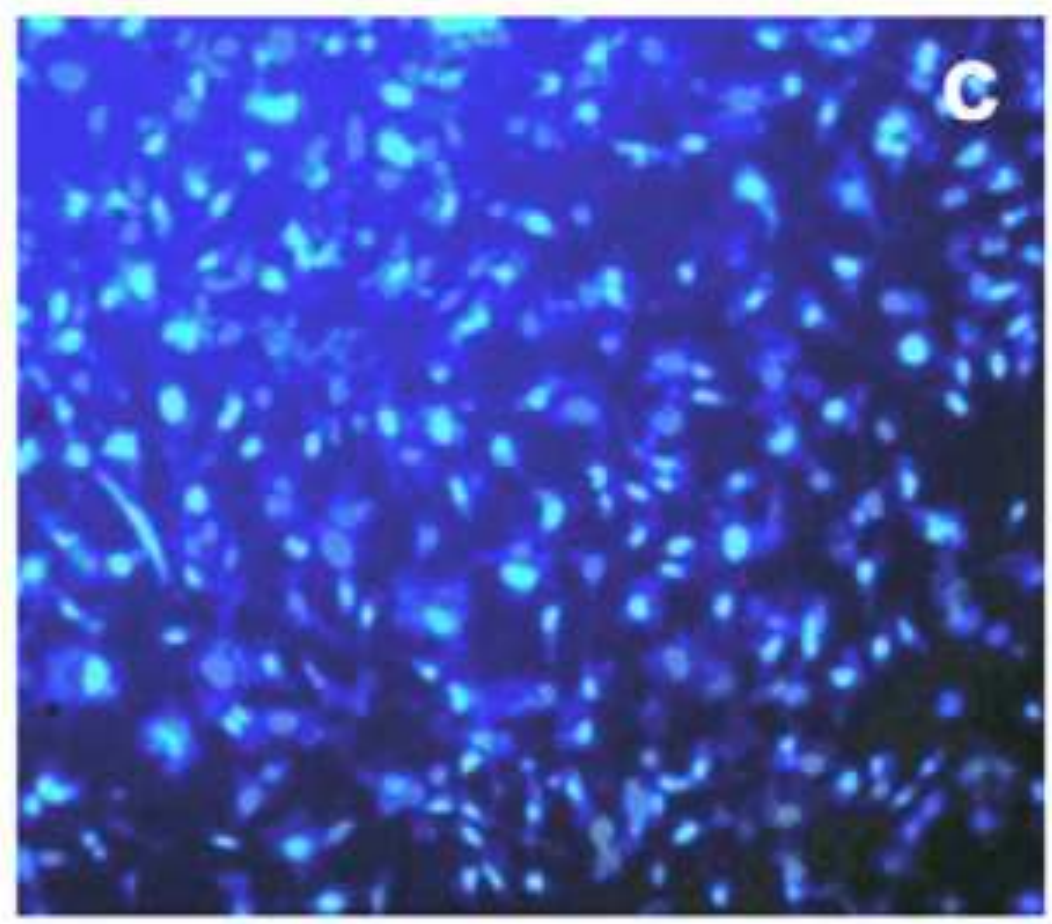




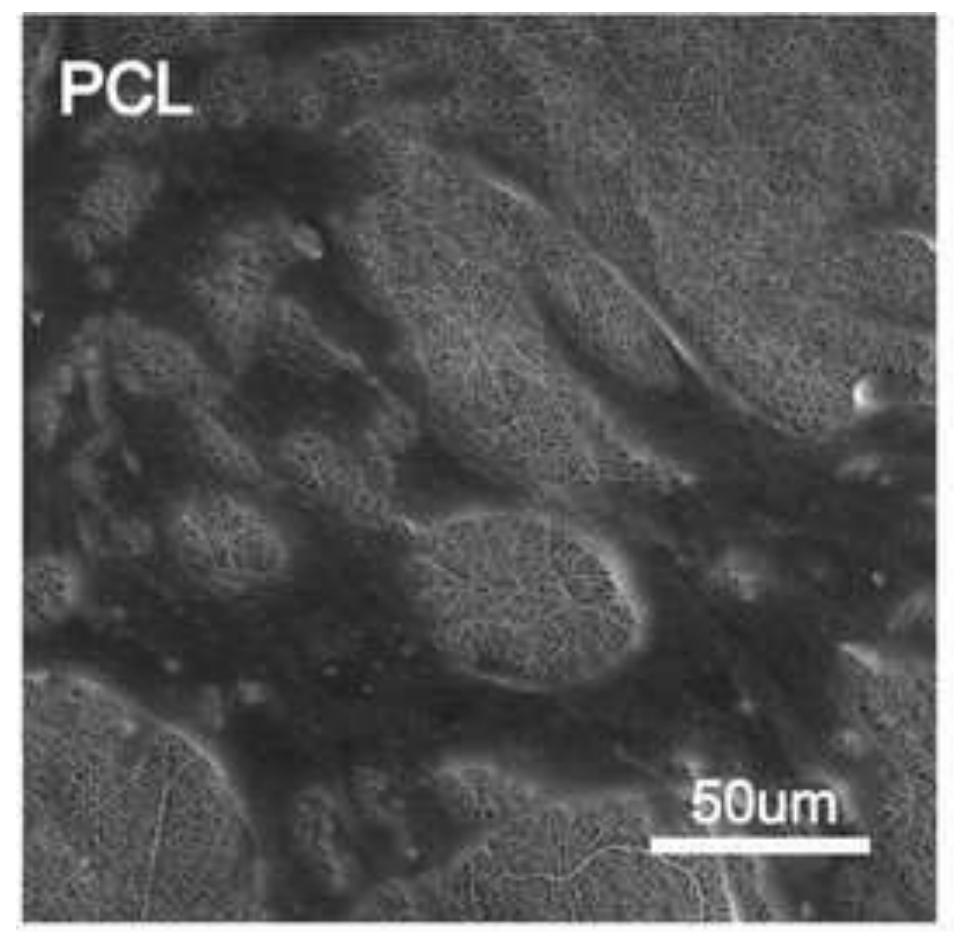

\section{PCLHAP}

50um

\section{PCLPCL-g-HAp}
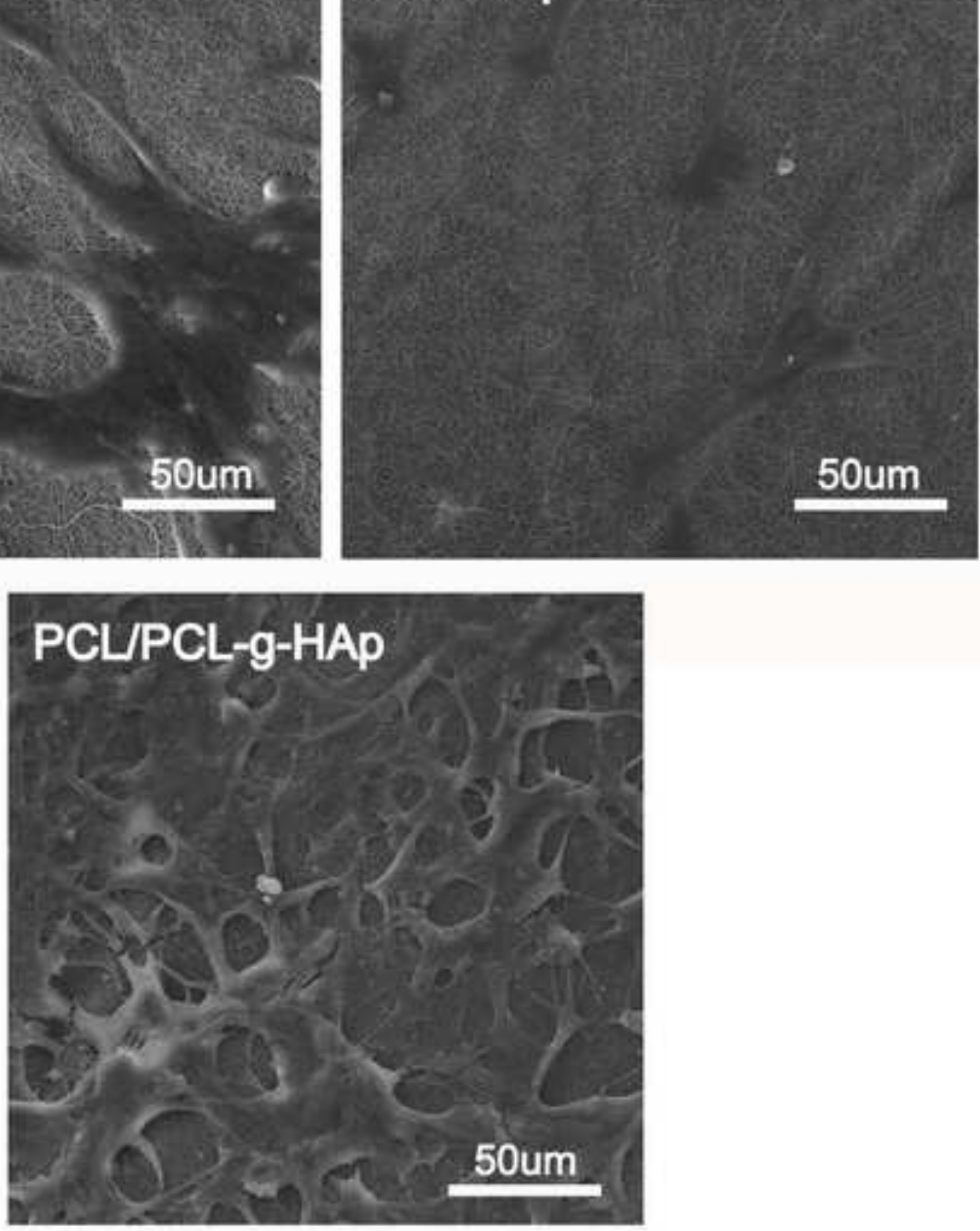

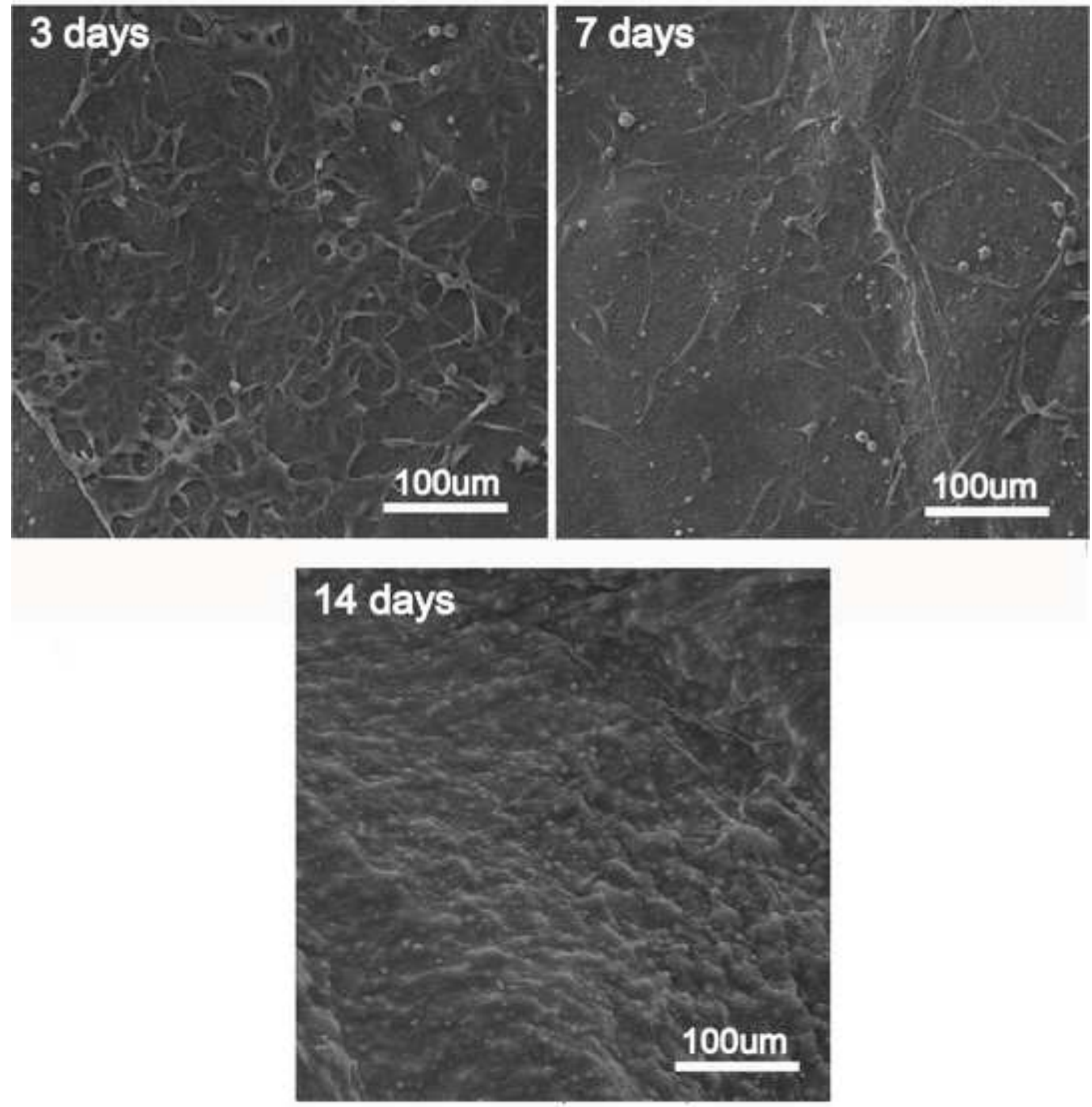

Figure 9 

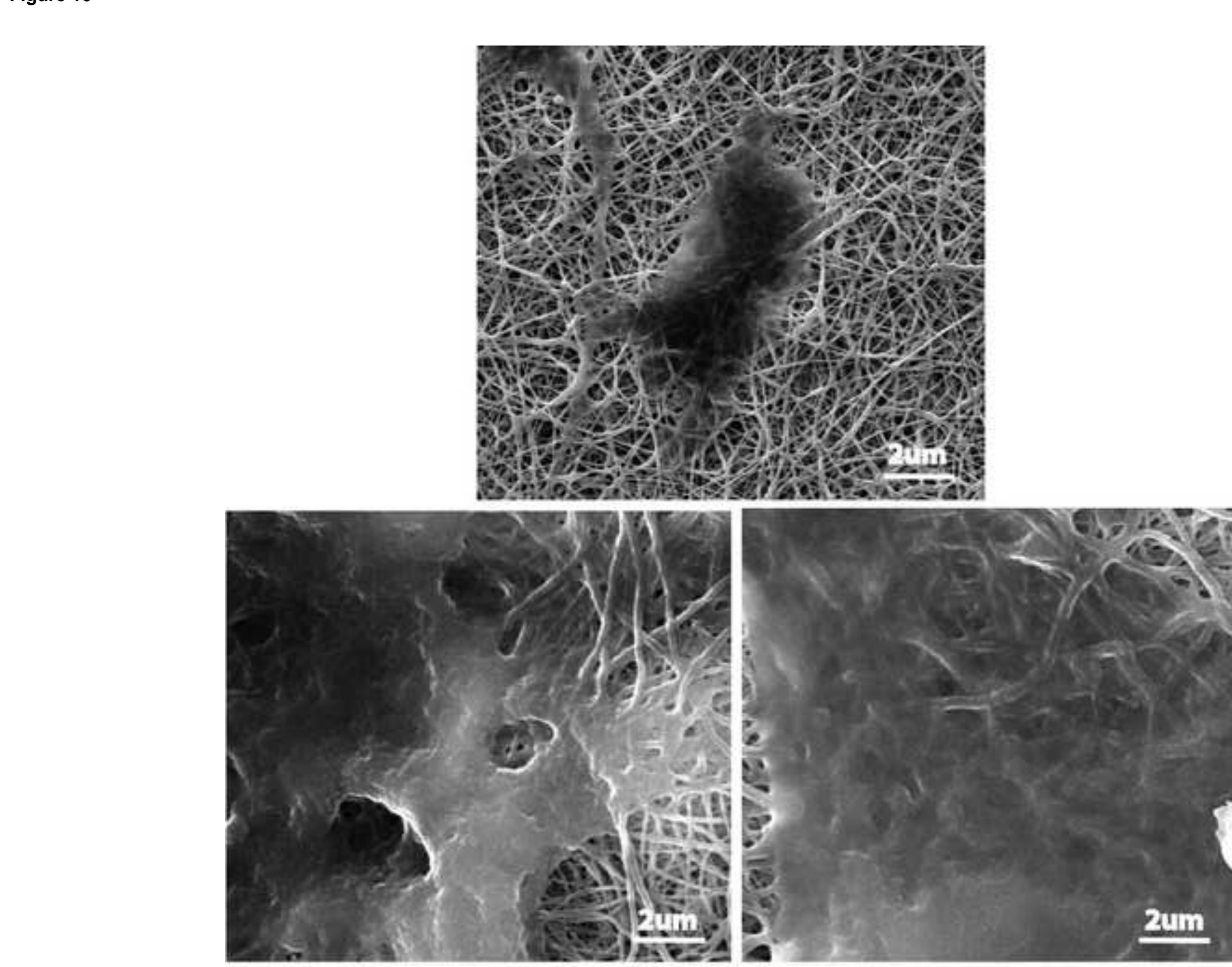

2 um

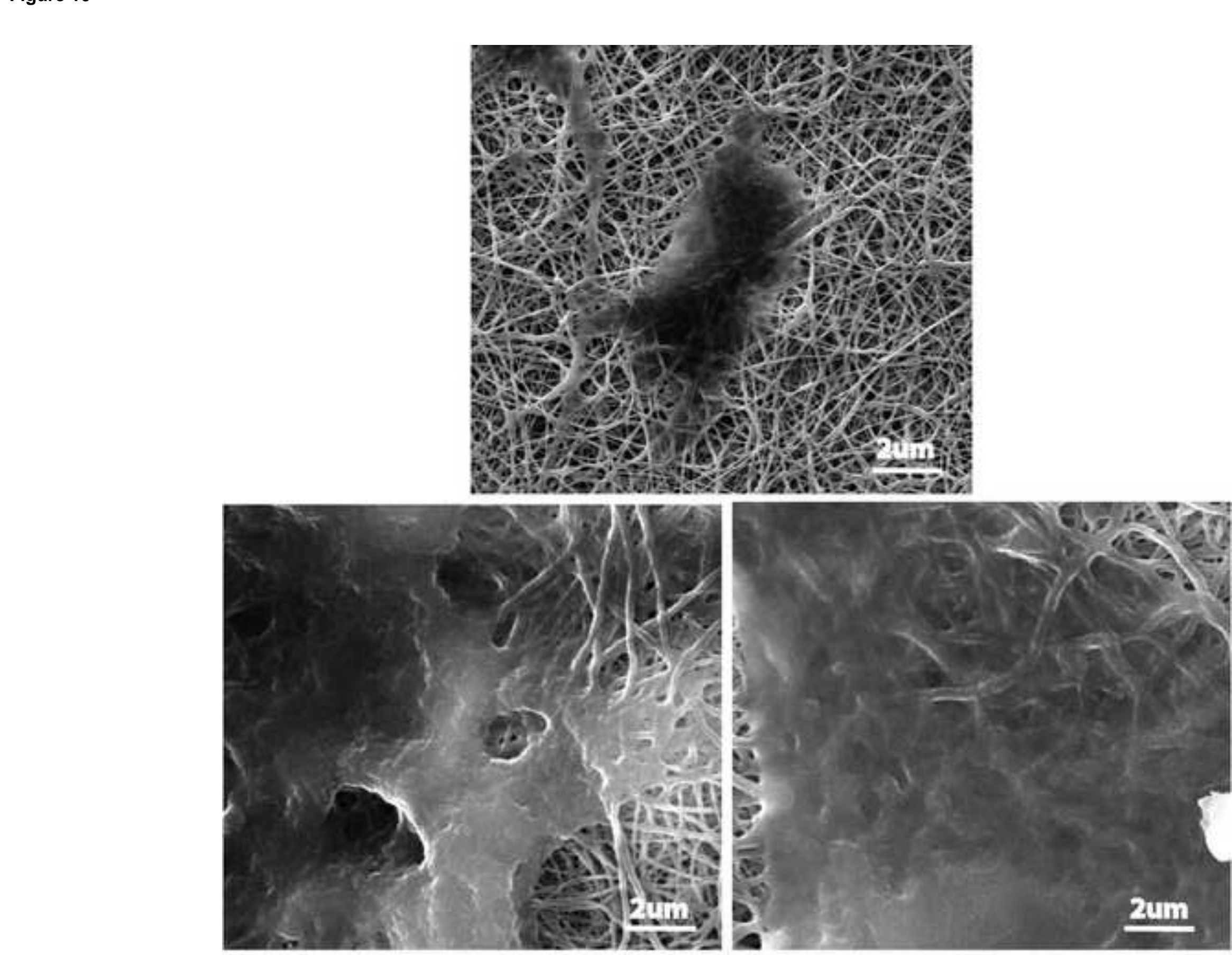




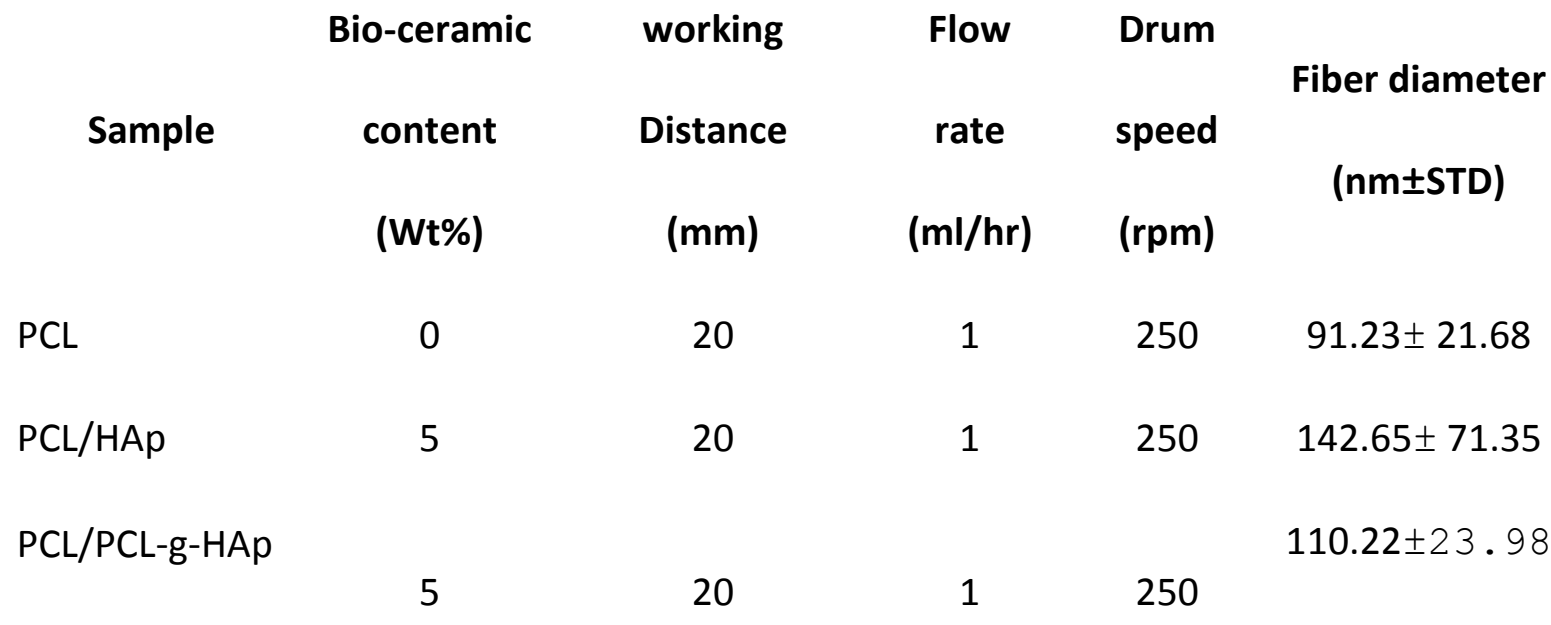


Film (degrees)
Water contact angle (degrees)

$\begin{array}{lllr}\text { PCL } & 80 & \text { PCL } & 118 \\ \text { PCL/HAp (5wt\%) } & 72 & \text { PCL/HAp (5wt\%) } & 107 \\ \text { PCL/PCL-g-HAp (5wt\%) } & 77 & \text { PCL/PCL-g-HAp (5wt\%) } & 110\end{array}$

